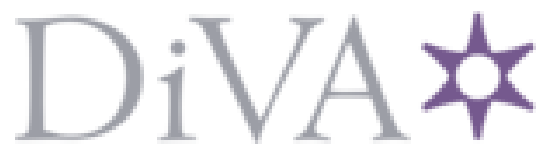

http://www.diva-portal.org

This is the published version of a paper presented at 14th International Conference, EANN 2013, Halkidiki, Greece, September 13-16, 2013.

Citation for the original published paper:

Gelzinis, A., Verikas, A., Bacauskiene, M., Vaiciukynas, E. (2013)

Learning Accurate Active Contours.

In: Lazaros Iliadis, Harris Papadopoulos \& Chrisina Jayne (ed.), Engineering

Applications of Neural Networks: 14th International Conference, EANN 2013,

Halkidiki, Greece, September 13-16, 2013 Proceedings, Part I (pp. 396-405). Berlin

Heidelberg: Springer Berlin/Heidelberg

Communications in Computer and Information Science

https://doi.org/10.1007/978-3-642-41013-0_41

N.B. When citing this work, cite the original published paper.

Permanent link to this version:

http://urn.kb.se/resolve?urn=urn:nbn:se:hh:diva-24042 


\title{
Learning Accurate Active Contours
}

\author{
Adas Gelzinis $^{1, \star}$, Antanas Verikas ${ }^{1,2}$, \\ Marija Bacauskiene ${ }^{1}$, and Evaldas Vaiciukynas ${ }^{1}$ \\ 1 Department of Electrical \& Control Equipment, \\ Kaunas University of Technology, \\ Studentu 50, LT-51368, Kaunas, Lithuania \\ adas.gelzinis@ktu.lt \\ 2 CAISR, Halmstad University, Box 823, S-30118 Halmstad, Sweden
}

\begin{abstract}
Focus of research in Active contour models (ACM) area is mainly on development of various energy functions based on physical intuition. In this work, instead of designing a new energy function, we generate a multitude of contour candidates using various values of ACM parameters, assess their quality, and select the most suitable one for an object at hand. A random forest is trained to make contour quality assessments. We demonstrate experimentally superiority of the developed technique over three known algorithms in the P. minimum cells detection task solved via segmentation of phytoplankton images.
\end{abstract}

Keywords: Active contour models, Energy function, Object detection, Image segmentation, Learning, Phytoplankton images.

\section{Introduction}

Active contour models (ACM) is one of the most successful techniques for object detection in images and have been widely used in image segmentation [1 3], analysis of medical images [4], analysis of protein spots in two-dimensional gel electrophoresis images [5], and many other tasks. ACMs are based on an energy minimization approach and allow incorporating a priori knowledge. To extract the desired object, ACM aims at evolving a curve, under some constrains, to fit the object.

Thus, ACM is a curve $\mathbf{c}(s)=[x(s), y(s)], s \in[0,1]$ deforming on the image region, to attain desired properties. Curve deformation is achieved by minimizing an energy function, for example:

$$
E(\mathbf{c})=\int_{0}^{1}\left[\frac{1}{2}\left(\alpha\left\|\mathbf{c}^{\prime}(s)\right\|^{2}+\beta\left\|\mathbf{c}^{\prime \prime}(s)\right\|^{2}\right)+E_{\text {ext }}[\mathbf{c}(s), f(I)]\right] d s
$$

where $\mathbf{c}^{\prime}(s)$ and $\mathbf{c}^{\prime \prime}(s)$ are the first and the second derivative of $\mathbf{c}(s)$ with respect to $s, \alpha$ and $\beta$ are parameters, and $E_{\text {ext }}$ stands for external energy linking the contour $\mathbf{c}(s)$ to specific features $f(I)$ of the image $I$. Both static (depending on

\footnotetext{
* Corresponding author.
}

L. Iliadis, H. Papadopoulos, and C. Jayne (Eds.): EANN 2013, Part I, CCIS 383, pp. 396-405, 2013. (C) Springer-Verlag Berlin Heidelberg 2013 
the image) and dynamic external forces (depending on the contour) have been used [6]. Static forces can be evaluated using image region intensity and texture information [7]. The following are two typical external energy functionals used to place a contour at edges [1] :

$$
\begin{aligned}
& E_{\text {ext }}(x, y)=-\|\nabla I(x, y)\|^{2} \\
& E_{\text {ext }}(x, y)=-\left\|\nabla\left[G_{\sigma}(x, y) * I(x, y)\right]\right\|^{2}
\end{aligned}
$$

where $\nabla$ is the gradient operator, $G_{\sigma}(x, y)$ is a two-dimensional Gaussian of standard deviation $\sigma$, and $*$ stands for the convolution operation.

Edge-based [1, 8] and region-based [2, 9] models are distinguished, depending on constraints applied. Edge-based models suffer from nearby adjacent objects and strong edges inside an object being searched, since they act as attraction sources for the active contour. Region-based models use statistical information inside and outside the contour and, for images with weak edges, usually perform better than edge-based models. ACM proposed by Chan and Vese is a popular representative ( $\mathrm{C}-\mathrm{V}$ method) of region-based models [2]. The $\mathrm{C}-\mathrm{V}$ model as well as some other popular ACMs assume constant intensity in various image regions [2]. Numerous techniques have been suggested to combine edge-based and region-based models [10].

The literature analysis indicates that focus of research in ACM area is on development of various energy functions based on physical intuition. Despite the vast number of energy functions used, all techniques experience difficulties in noisy environment. It is hard to succeed in designing a "universal" energy function, capable of dealing with large data variations.

In this work, instead of designing a new energy function, we generate a multitude of contour candidates, assess their quality, and select the most suitable one for an object at hand. Also, aiming to provide the edge-based ACM with rich and diverse information on image edges, we focus on image preprocessing. The energy function used in this work is given by equations (11), (2), and (3).

\section{Data}

Accurate detection of Prorocentrum minimum P. minimum cells in phytoplankton images was the main motivation to develop a new contour detection technique. Fig. 1 presents two phytoplankton images with $P$. minimum cells. Images for the analysis were obtained from a simple RGB colour camera of $3264 \times 2448$ pixels attached to an inverted microscope with magnification of 400x. Only $G$ image component has been used in this study, since the other two components do not provide much new information for contour detection.

The $P$. minimum species is known to cause harmful blooms in many estuarine and coastal environments. There is a hypothesis that $P$. minimum cells gradually change their shape when adapting to adverse biotic (increased virus pressure) conditions. To study shape changes of $P$. minimum cells, accurate cell detection is required. The length of $P$. minimum cells varies from 14 to $22 \mu \mathrm{m}$, while the width range from 12 to $18 \mu \mathrm{m}$. 
Presence of chlorophyll in phytoplankton cells makes them glow under UV light and this property becomes of immense help for detecting P. minimum cells. Two consecutive, with a delay of a few seconds, photos (using light and fluorescence microscopy) were obtained of the same location, see Figure 1 .
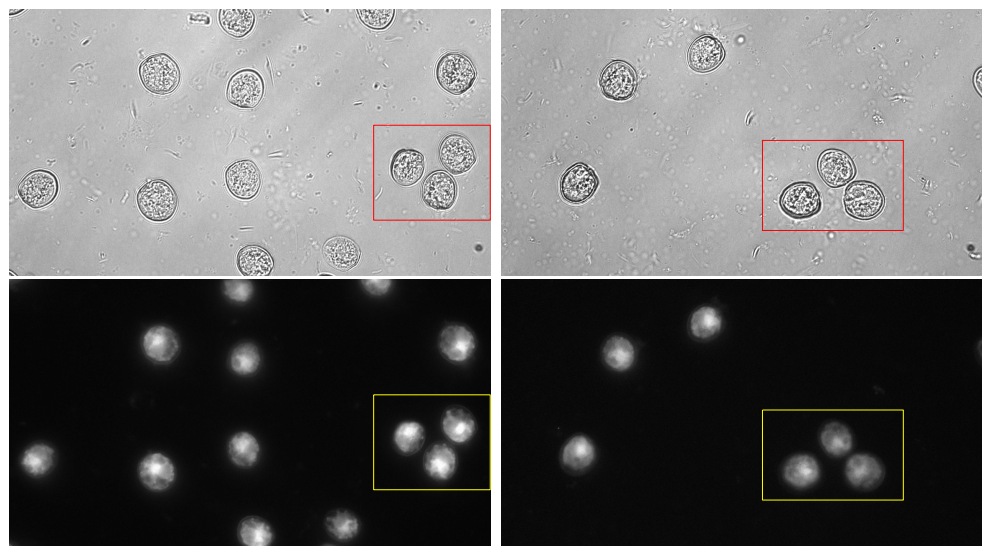

Fig. 1. Two examples of phytoplankton images with P. minimum cells (top); phytoplankton fluorescence images of the same location (bottom).

\section{Contours of Cells}

Images from both the light microscopy and fluorescence microscopy are used to find cells and their center coordinates. Regions of interest are found by simple thresholding [11] of images from the fluorescence microscopy, while the phase congruency-based technique [12] is used to find the center coordinates of objects (cells). Only cells detected in both fluorescence and light microscopy images are used for further analysis.

\subsection{Generating a Multitude of Contour Candidates}

A $2 \mathrm{D}$ shell is a characteristic feature of P. minimum cells. We rely on this fact by computing a local intensity gradient in the direction of cell center (see Section 3.2) and use this gradient image to generate a multitude of contour candidates (12 for each cell) by varying the parameters $\alpha$ and $\beta$ of the ACM, see Eq. 1. A circle with a center point coinciding with the cell center and a radius exceeding the maximal possible cell radius is selected as initial contour. Parameters $\alpha$ and $\beta$ control the stretch and stiffness of the contour. Optimal values of $\alpha$ and $\beta$ parameters are different for different cells due to many factors, such as: presence of other nearby objects, clarity of cell contour, focussing accuracy, and others. We believe that the multitude of contour candidates generated using different values of the parameters $\alpha$ and $\beta$, contains a contour coinciding well with borders of the cell being studied. 


\subsection{Local Intensity Gradient in the Direction of Cell Center}

To obtain the gradient, a cell image along with surrounding areas is transformed to the polar coordinate system and the local intensity gradient is computed along the direction of radius. The obtained gradient image is then transformed back to the original coordinate system and used by the ACM. Fig. 2 provides some insights into the gradient. Each of the three images shown in Fig. 2 presents results of gradient computation in the direction of a different cell center. The cell center used to compute the gradient is shown by a cross. As can be seen, the cell, in the direction of which center the gradient is computed, manifests itself by a much clear cell boundary if compared to the other cells. It is worth noting rather small influence of the nearby objects on the gradient computation results.
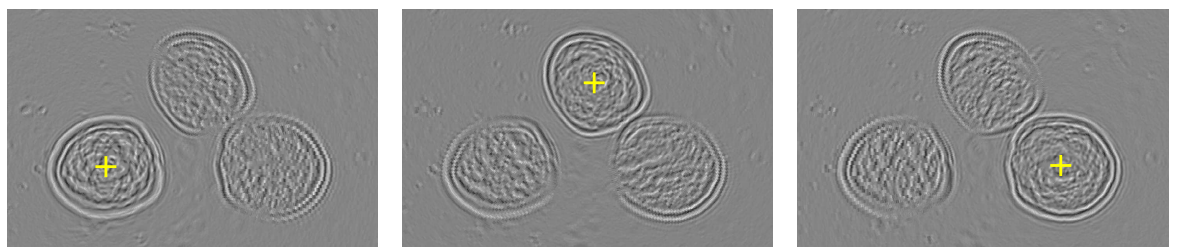

Fig. 2. Images of local intensity gradient in the direction of cell center

\section{Assessing Quality of Cell Contours}

A random forest (RF) [13] is trained to provide the quality assessment of cell contours. A training set of labelled data (contours) is required to build a random forest. An expert performs the labelling by assigning one of the following five labels to each contour from the training set: a) unappropriate, b) bad, c) satisfactory, d) good, and e) excellent.

Many factors influence the quality of a generated contour: clarity and complexity of the cell border, the image quality, accuracy of focussing, nearby objects, and cell texture. Therefore, properties of both the contour curve and image region where the curve is placed are considered, to assess the contour quality.

\subsection{Features Used to Characterize Contours}

The following four groups of features are used to characterize contours of cells: i) Object geometry; ii) Fourier descriptors; iii) Contour curvature; and iv) Image properties in the vicinity of the contour.

The object geometry features, Fourier descriptors, and the contour curvature features characterize object and contour curve properties, while features of the fourth type reflect image properties in the vicinity of the contour. 


\section{Object Geometry Features}

We adopted the following features to characterize object geometry: i) Area; ii) Perimeter; iii) Major Axis; iv) Eccentricity; v) Circularity; vi) Roundness.

\section{Fourier Descriptors}

A $K$-point digital boundary in the $x y$-plane can be represented as the sequence of coordinates $c(k)=[x(k), y(k)]$, for $k=0,1, \ldots, K-1$. Each coordinate pair can be treated as a complex number so that $c(k)=x(k)+j y(k)$. The discrete Fourier transform of $\mathrm{c}(\mathrm{k})$ is

$$
a(u)=\frac{1}{K} \sum_{k=0}^{K-1} c(k) e^{-j 2 \pi u k / K}
$$

for $u=0,1, \ldots, K-1$. The complex coefficients $a(u)$ are called the Fourier descriptors of the boundary. Instead of using all the Fourier descriptors, only the absolute values of the first $P<K$ coefficients were used.

\section{Curvature Features}

For a plane curve given as $c(k)=[x(k), y(k)]$, the curvature is given by

$$
\kappa(k)=\frac{\left|x^{\prime} y^{\prime \prime}-x^{\prime \prime} y^{\prime}\right|}{\left(x^{\prime 2}+y^{\prime 2}\right)^{3 / 2}}
$$

where primes refer to derivatives with respect to $k$.

Based on the curvature assessment at each contour point, we compute four curvature related features: $(1) \operatorname{mean}(\kappa) ;(2) \max (\kappa) ;(3) \operatorname{std}(\kappa)$; and (4) upper quartile value of $\kappa$.

\section{Image Features}

For each contour $c(k)$ point $k=1,2, \ldots, K$, one interior $c_{\text {int }}(k)$ and one exterior $c_{\text {ext }}(k)$ point are chosen perpendicular to the contour at a distance $\delta$. Various image intensity characteristics on both sides of the contour are computed based on positions of $c_{i n t}(k)$ and $c_{\text {ext }}(k)$. Average image gradient as well as average image gradient in the vicinity of the contour are also used as image features: (1) standard deviation of the intensity difference between the interior and exterior points: $\operatorname{std}\left(I_{c_{\text {int }}}-I_{c_{\text {ext }}}\right) ;(2-3)$ mean and standard deviation of intensity of the interior and exterior points: mean $\left(I_{c_{\text {int }}}\right), \operatorname{std}\left(I_{c_{\text {int }}}\right)$; (4-5) mean intensity and standard deviation of contour points: mean $\left(I_{c}\right), \operatorname{std}\left(I_{c}\right) ;(6-7)$ intensity mean and standard deviation of the exterior points: $\operatorname{mean}\left(I_{c_{e x t}}\right), \operatorname{std}\left(I_{c_{e x t}}\right)$; (8)average gradient magnitude: mean $\|\nabla I\| ;(9)$ average gradient magnitude in the vicinity of the contour: mean $\left(\left\|\nabla I_{c_{i n t}, c, c_{e x t}}\right\|\right)$.

\subsection{Assessing Contour Quality by Experts - Labelling Contours}

Twelve contours were generated for each cell using different parameters of the ACM algorithm. Different background, varying clarity of cell borders, other objects inside and outside cells are the factors strongly affecting results of automatic contour detection. It is a rather hard task for the experts to decide where 
exactly the actual cell contour should be drawn. To draw exact contours for a large number of cells is a very tedious task. Therefore, instead of drawing exact cell contours, three experts were asked to assess quality of automatically generated contours. We used five contour quality grades: Unappropriate (1), Bad (2), Satisfactory (3), Good (4), and Excellent (5).

Fig. 3 presents four examples of contours of different quality. It is worth noting that, for some cells, amongst 12 contours generated by the algorithm for one cell, non the contours was deemed by the experts as being excellent, good or satisfactory, meaning that all 12 contours were deemed as being bad or unappropriate. Table 1 presents statistics of contour assessment, where the "All contours" columns show the percentage of all contours in different categories, as deemed by the experts. The rightmost column shows how the best contours, only one (best) contour for each cell, were assessed by the experts.
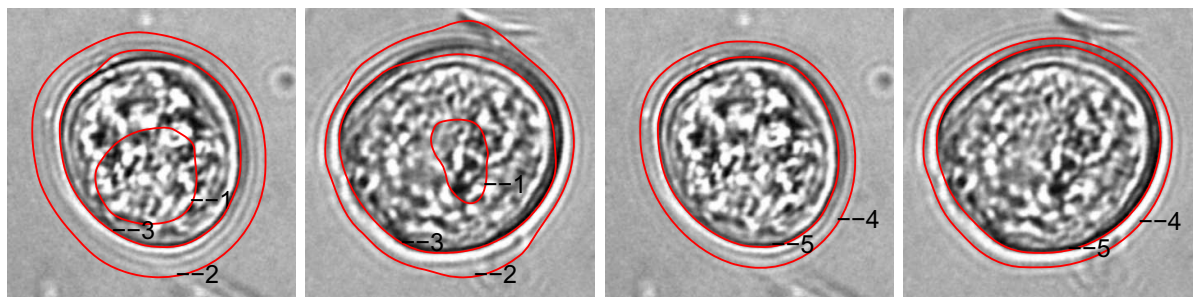

Fig. 3. Contours graded as Unappropriate, Bad and Satisfactory on the left, and Good and Excellent on the right

Table 1. Statistics of contour assessment by experts

\begin{tabular}{l|rr|rr}
\hline Grade & \multicolumn{2}{|c|}{ All contours } & \multicolumn{2}{c}{ Best contours } \\
& \#N & $\%$ & \# N & $\%$ \\
\hline Excellent & 710 & 22.9 & 200 & 77.5 \\
Good & 324 & 10.5 & 35 & 13.6 \\
Satisfactory & 487 & 15.7 & 21 & 8.1 \\
Bad & 358 & 11.6 & 2 & 0.8 \\
Unappropriate & 1217 & 39.3 & 0 & 0.0 \\
\hline
\end{tabular}

\section{Experimental Investigations}

The following issues, which are of key importance for the success of the algorithm, have been studied experimentally: i) accuracy of automatic contour quality assessment; ii) quality of selected ("best") contour, ability to detect a selected contour of bad quality; iii) optimal values of ACM parameter. The study confirms that to generate a contour of good quality, different ACM parameters are needed for different cells even in images of the same type; iv) comparison of the proposed contour detection technique with other methods. 


\subsection{Accuracy of Automatic Contour Quality Assessment}

For each of 258 cells used in the experiments, 12 contours were generated using different values of $\alpha$ and $\beta$ parameters, see Eq.(11). In this experiment, we set $\alpha=\beta$ and varied their values in the interval [0.02 0.25]. Quality of all generated contours was assessed by three experts, giving grades from 1 to 5 , as it has been discussed in Section 4.2. These contour quality grades together with equally weighted contour features were used to train RF for automatic contour quality assessment.

To evaluate accuracy of the automatic contour quality assessment, the out-ofbag set of contours was used. The average mean squared error of contour quality evaluations given by the experts and the model was equal to $M S E=0.16$. When quality prediction values were rounded off to the five discrete grades, discrepancy between grades given by the experts and the model were observed for $17.9 \%$ of contours. Bearing in mind the fact that the experts often disagreed when giving grades Good, Satisfactory and Bad, the obtained results are very encouraging.

\subsection{Quality of Selected Contours}

The following are important issues to consider when evaluating robustness of the developed model for automatic contour quality assessment. Is the automatically selected contour the best amongst all generated? How often the automatically selected contour is the best amongst all generated? To address these issues, an automatic assessment was deemed to be erroneous, if an automatically selected "best" contour was different from the one that obtained a highest grade from the experts for that particular cell. There were only 13 such cells out of 258 available (about $5.0 \%$ of errors).

The experiment was repeated using new contours generated by varying $\alpha$ and $\beta$. To increase the variety of new unseen contours, 36 contours were generated for each cell. To assess quality of the contours, RF trained in the previous experiment was used. Since the number of contours used in this experiment was rather large, the experts assessed only if the RF failed to select the best available contour for each cell. There were 7 such errors, out of 258 possible, i.e., for 7 cells, a contour selected by the RF, was not the best according to the experts.

Table 2 presents quality of best cell contours according the experts. In this table, the contour categories Bad and Unappropriate were combined into one, Unappropriate. The results presented in Table 2 slightly differ from those given in Table 1. The discrepancy is due to the fact that, the best contour for each cell was selected from a larger set in this experiment (36 instead of 12). Moreover, since in this experiment the experts were asked to select and assess only one, best, contour for each cell, the assessments happened to be more rigorous compared to the assessments used to train the RF. As can be seen from Table2, more than $92 \%$ of all contours are of excellent and good quality.

It is important to detect contours of bad quality and eliminate them from further analysis. The experiments have shown that most of automatically selected contours deemed by the experts as being unappropriate or satisfactory, 
Table 2. Assessment of the best cell contours, one for each cell

\begin{tabular}{lrr}
\hline Grade & \# N & $\%$ \\
\hline Excellent & 198 & 76.8 \\
Good & 40 & 15.5 \\
Satisfactory & 13 & 5.0 \\
Unappropriate & 7 & 2.7 \\
\hline
\end{tabular}

obtained low grades from the automatic evaluation too. To eliminate contours of low quality, an experimentally selected threshold value equal to 3.2 was applied. The thresholding eliminated $9 \%$ of lowest quality contours. To this group were automatically attributed all contours deemed by the experts as being of unappropriate or satisfactory quality. Only one contour of satisfactory quality according to the experts obtained rather high quality value, equal to 4.5, from the RF. Four contours of good quality according to the experts were automatically assigned to the group of bad quality contours.

The experimental investigations show that the best contour is selected from the set of contours generated for one cell with $95 \%$ accuracy. Detection accuracy of contours of satisfactory and bad quality exceeds $98 \%$ ( 5 errors). After the automatic elimination of bad quality contours (about $9 \%$ ), only one contour of satisfactory quality was found in the group of good quality contours.

\subsection{Optimal Values of ACM Parameters}

For a given cell, ACM parameter values leading to contours of the highest grade are called optimal. In this experiment, the assessment was done automatically using the trained RF. Fig. 4 displays the histogram of $\alpha$ and $\beta$ values (in this case, $\alpha=\beta$ ) leading to the best cell contours. Observe that values of the ACM parameters were varied in a broad range: from values hindering the ACM from placing a contour sufficiently close to an actual cell contour to values placing the contour inside the cell. The distribution confirms that optimal $\alpha$ and $\beta$ vary in a broad range, and different ACM parameter values are optimal for different cells.

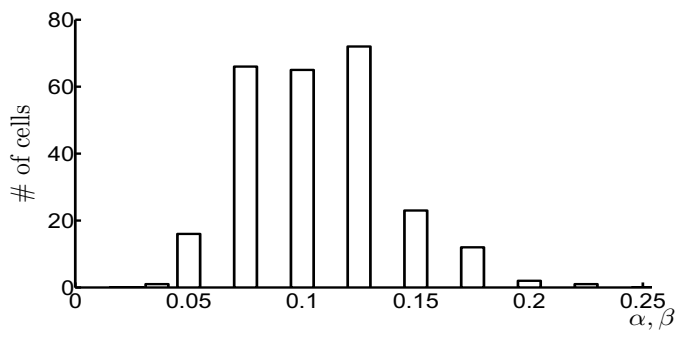

Fig. 4. The histogram of $\alpha$ and $\beta$ values leading to the best cell contours 


\subsection{Comparison with Other Methods}

The technique was compared with the $\mathrm{C}-\mathrm{V}$ method [2], and two recently developed algorithms [3, 14]. All the algorithms have several adjustable parameters. Values of the parameters were carefully selected separately for each algorithm.

In total, 49 phytoplankton images containing $258 \mathrm{P}$. minimum cells were processed to test the algorithms. The segmentation results were evaluated by the experts and are summarized in Table 3. Observe that in this experiment, only contours assigned to the Excellent and Good groups were considered as being correct. As can be seen from Table 3 , the proposed technique provided the best performance. Segmentation by the algorithms used for the comparison often resulted either in over-segmentation or under-segmentation (leakages of contour and inability to separate several nearby cells).

Table 3. Summary of cell contour detection results

\begin{tabular}{lrr}
\hline Algorithm & Correct & Accuracy, \% \\
\hline Bernard [3] & 42 & 16.28 \\
Chan \& Vese [2] & 52 & 20.16 \\
Shi [14] & 152 & 58.91 \\
Proposed & 238 & 92.25 \\
\hline
\end{tabular}

\section{Discussion and Conclusions}

Rather than focusing on constructing an energy function based on physical intuition, as many recent studies of ACMs do, we advocate the idea of generating a multitude of contour candidates, assessing their quality, and selecting the most suitable one for an object at hand. We experimentally demonstrate the superiority of the developed approach over three well known ACM algorithms applied to cell segmentation in the microscopy imagery task. Sensitivity to nearby objects and strong edges inside a target object is highly reduced and segmentation becomes more noise-robust using the proposed approach.

The experimental investigations have shown that in $92.25 \%$ of cases, contours of $P$. minimum cells were accurately determined. For each cell tested, the proposed algorithm was able to select the best contour from a set of generated in $95 \%$ of cases. In the remaining $5 \%$ of cases, contours selected by the algorithm were only slightly worse than the best possible. Cases where non of generated contours was appropriate for a given cell were automatically detected with higher than $98 \%$ accuracy. By setting an appropriate, experimentally selected, threshold value on predicted contour quality, $9 \%$ of contours were categorized as being of unappropriate quality and eliminated from further analysis. Amongst the remaining contours only one contour attributed by the experts to the Satisfactory group, was assigned to the Good group by the algorithm. The investigations have shown that the developed technique provides contour quality assessment with high accuracy and can be used for automatic contour quality evaluation and contour selection. 
Acknowledgement. This research was funded by a grant (No. LEK-09/2012) from the Research Council of Lithuania.

\section{References}

1. Kass, M., Witkin, A.P., Terzopoulos, D.: Snakes: Active contour models. International Journal of Computer Vision 1(4), 321-331 (1988)

2. Chan, T., Vese, L.: Active contours without edges. IEEE Transactions Image Processing 10(2), 266-277 (2001)

3. Bernard, O., Friboulet, D., Thevenaz, P., Unser, M.: Variational B-spline level-set: A linear filtering approach for fast deformable model evolution. IEEE Transactions on Image Processing 18(6), 1179-1191 (2009)

4. Shang, Y., Yang, X., Zhu, L., Deklerck, R., Nyssen, E.: Region competition based active contour for medical object extraction. Computerized Medical Imaging and Graphics 32(2), 109-117 (2008)

5. Savelonas, M.A., Mylona, E.A., Maroulis, D.: Unsupervised 2D gel electrophoresis image segmentation based on active contours. Pattern Recognition 45(2), 720-731 (2012)

6. Veronese, E., Stramare, R., Campion, A., Raffeiner, B., Beltrame, V., Scagliori, E., Coran, A., Ciprian, L., Fiocco, U., Grisan, E.: Improved detection of synovial boundaries in ultrasound examination by using a cascade of active-contours. Medical Engineering \& Physics (2012), doi.org/10.1016/j.medengphy.2012.04.014

7. Chakraborty, A., Staib, L., Duncan, J.: Deformable boundary finding in medical images by integrating gradient and region information. IEEE Transactions Medical Imaging 15(6), 859-870 (1996)

8. Caselles, V., Kimmel, R., Sapiro, G.: Geodesic active contours. International Journal of Computer Vision 22(1), 61-79 (1997)

9. Ronfard, R.: Region-based strategies for active contour models. International Journal of Computer Vision 13(2), 229-251 (1994)

10. Tao, W., Tai, X.C.: Multiple piecewise constant with geodesic active contours (MPC-GAC) framework for interactive image segmentation using graph cut optimization. Image and Vision Computing 29, 499-508 (2011)

11. Otsu, N.: A threshold selection method from gray-level histograms. IEEE Trans Systems Man \& Cybernetics 9(1), 62-66 (1979)

12. Verikas, A., Gelzinis, A., Bacauskiene, M.: Phase congruency-based detection of circular objects applied to analysis of phytoplankton images. Pattern Recognition 45(4), 1659-1670 (2012)

13. Breiman, L.: Random forests. Machine Learning 45, 5-32 (2001)

14. Shi, Y., Karl, W.C.: A real-time algorithm for the approximation of level-set-based curve evolution. IEEE Transactions on Image Processing 17(5), 645-656 (2008) 